\title{
Role of 3-Y markers in Y deleted allele on amelogenin in autosomal STR
}

\begin{abstract}
In forensic investigation, DNA plays a vital role to link the suspect/individual, victim and the scene of occurrence. DNA constitutes the genetic material of an individual and offers the preparation of DNA profile from the recovered biological materials often in sexual assault cases. This leads to apprehend the perpetrator of any crime based on the obtained DNA profile from such biological samples. It helps to identify the gender of an individual in missing identity and also in mixed samples. Structural polymorphism, which is rapidly being determined as the utmost form of genome variation is predominantly found on the $\mathrm{Y}$ chromosome. The gender from any sample is always determined by the DNA profile based on the presence of $\mathrm{Y}$ allele in amelogenin in autosomal STR in single marker of $Y$ chromosome. The detection of any single $\mathrm{Y}$ chromosome in an unknown sample proves that, it belongs to a male individual. But in a few instances, it becomes a challenging task whenever due to mutation, no Y allele on amelogenin in male DNA profile in autosomal STR. In our previous study, we used the Identifiler plus Kit in the population of Delhi and observed that the profile of 708 samples was successfully obtained while only 2 profiles were observed without $\mathrm{Y}$ allele on amelogenin marker. The above two $\mathrm{Y}$ deleted samples were amplified by use of Globalfiler kit having 3 markers of Y chromosome. In such cases, Globalfiler kit helps to provide the presence of two other markers of Y chromosomes for determination of male gender of sample.
\end{abstract}

Keywords: genetic material, gender, amelogenin, mutation, Y chromosome, autosomal
Volume 4 Issue 2 - 2019

\author{
Naresh Kumar,' Amit Chauhan, ${ }^{2}$ Ritika \\ Gupta,' SK Shukla ${ }^{2}$ \\ 'Forensic Science Laboratory, Home Department, Govt NCT \\ of Delhi, India \\ ${ }^{2}$ Amity Institute of Forensic Sciences, Amity University, India
}

Correspondence: Naresh Kumar, Forensic Science Laboratory, Home Department, Govt. NCT of Delhi, Rohini, Delhi, India, Tel +91-9971918173, Email nareshkumar2982@yahoo.com

Received: February 27, 2019 | Published: April 22, 2019

\section{Introduction}

Now a day, people from various parts of country are moving towards the metropolitan cities for their livelihood, better facilities. The people who migrate in Delhi for their livelihood range more than 15 million. That's why, the crime rates are increasing day by day along with the high rate of population. Due to the socio-economic reasons, education level of people, crime including sexual assault, eve-teasing etc. are increasing very rapidly. The law enforcement agencies are taking strict actions against the perpetrator to prevent from these crimes. To follow the international standards for justified examination of evidences, various types of evidences play a vital role to solve a crime. A systematic procedure of collection the evidences, as per the law of scientific evidences are followed to punish the actual culprit of any crime. In a few instances, accused are acquitted by the courts in light of inappropriate examination of samples or not following the guideline of international standard. The cases of sexual assault and missing identity, the samples are sent to the forensic science laboratories to determine the gender and identify the individual based on DNA profiles. In such cases, where the gender of sample is not known to the experts and a very high amount of female biological material is present; examination of samples becomes a necessity. In case the examination is conducted by untrained and inexperienced scientific staff, it makes it vulnerable to surrender before the court or defence counsel. It is necessary to examine the samples in proper way and report the cases with proper justification. It has been observed so many times in sexual assault cases/rape that a mixed profile of male and female samples recovered from vaginal swab or undergarments.
But due to the small quantity of male fraction and missing of Y allele on marker amelogenin, the results were misinterpreted as negative case. ${ }^{1}$ It has been proved so many times that $\mathrm{Y}$ chromosomes provide the gender discrimination. Deterioration of Y chromosome has started since long back and its size was similar to as $\mathrm{X}$ chromosomes. The human amelogenin has two homologous alleles one on $\mathrm{X}$ and other on $\mathrm{Y}$ chromosome. The size of Amelogenin X and amelogenin Y differ in size, $\mathrm{X}$ gene size is 2872 base pairs and $\mathrm{Y}$ is 3272 base pairs. Location of amelogenin on $\mathrm{X}$ is Xp 22.1-22.3 and $\mathrm{Y}$ is Yp11.2. ${ }^{2}$

Utilization of nano drop has become very common in forensic investigation for the quantitation of DNA; even now it has started for the quantitation of all samples. Degraded samples give the appropriate quantity due to the presence of degraded DNA. Even Y filer kit is not being used in such cases and reports are deposited only based on autosomal STR. The identification of gender from a sample is always based of amelogenin marker. ${ }^{3}$ In case of sexual assault or unidentified or decomposed dead bodies, where $\mathrm{Y}$ allele is absent/ degenerated by use of Identifiler plus or power plex 21 kit. These kits contain one amelogenin marker and absence of $Y$ allele on amelogenin, sample treated as female in place of male.

It is absolutely right to obtain the Y profile to support the multiplex autosomal STR kit ${ }^{4}$ but it should be attempted as per different multiplex different kit available. Although it is very challenging in forensic case work but the ultimate purpose of amelogenin in DNA profile is to determine the gender of an individual/suspect. ${ }^{5}$ The detection of amelogenin and determination of gender can be done by quantitative PCR. In other methods, that are used for sex determination relating to 
$\mathrm{X}$ and $\mathrm{Y}$ which are found on centromeric regions of sex determining region (SRY) region avail in Quantifiler duo kit may also help in determination of species. In sexual assault cases, it has been observed that the quantity of male part is very truncated to the females. It occurs due to the delay and hygiene condition of victim than, it become more difficult to identify the $\mathrm{Y}$ on amelogenin. Here after, two possibilities outcomes either $\mathrm{Y}$ is not present in the sample because of, it is of a female or $\mathrm{Y}$ could not be detected due to genetic specificity of the sample. It is a fact that $\mathrm{Y}$ allele is not detected in few of male samples due to amelogenin ( $\mathrm{Y}$ null allele) from those kits that are only used amelogenin for sex determination. In this study, the blood samples were collected to generate the $\mathrm{Y}$ allele in such samples where quantity of Male DNA was $125 \mathrm{ng} / \mu 1$ detected through quantifiler duo kit Applied Bio systems. ${ }^{6}$ The Duo kit (applied Bio systems) is considered the best way and first step to decide whether a sample belongs to a male or female irrespective of the null AMELY male DNA samples. Moreover, quantity of DNA and inhibition of a sample will also be determined. Further, Y STR amplification will help to determine the gender of the sample and may strengthen the opinion that the particular sample is of a male. Therefore, it is suggested to use the Global filer kit which contains the 24 markers and also contains the 3 markers of $Y$ chromosomes.

\section{Material and methods}

The samples were received in the Forensic Science laboratory for analysis in sexual assault cases/ murder cases. During the analysis of these cases, out of 708 male samples; it was found that two male samples were failed to give $\mathrm{Y}$ alleles (AMELY) on amelogenin marker. ${ }^{5}$ All the samples were received and preserved at 4 degree Celsius for 2 to 3 months. All samples were further analysed for DNA isolation from the vaginal swabs and underwear of victim and matched with the blood samples of accused. A 3/4 portion of vaginal swab was cut into microcentrifuge tube of $1.5 \mathrm{ml}$. The samples were added in Forensic buffer, Proteinase K and SDS as per the recommendation of protocol. The samples were kept overnight for digestion of cell (female fraction). In sexual assault cases, the separation of female fraction is not fool proof technique, but it reduces the female part present in the samples. The samples were centrifuged the very next day and supernatant was discarded. The sperm (Male fraction) was left. The samples were again added in forensic buffer, Proteinase $\mathrm{K}$ and SDS and dithiothreitol to digest the spermatozoa as per the protocol. The samples where spermatozoa were present in enough quantity were collected for automate extraction Applied Bio systems as per manufacturer protocol.

\section{Real time PCR}

PCR technique was performed by using Quantifiler Duo kit of applied Bio system as per manufacture protocol. Total volume was $25 \mu 1$ that were having the reaction mix and primer and DNA sample as per protocol. Standards were used with the samples to observe the accuracy of results. These results help us to determine the availability of human and male DNA in the samples. This is reliable technique to determine the gender from forensic sample while amelogenin based technology is not fully reliable. Even this technique is useful in case of in prenatal diagnosis. ${ }^{7}$

Samples were diluted as per value given by RT-PCR and approximately $1 \mathrm{ng} / \mu \mathrm{l}$ DNA was used for sequencing the samples and Globalfiler kit was used for PCR amplification Applied Biosystem. ${ }^{6}$ This kit contains 24 markers including 3 markers for sex determination and was used for PCR amplification. PCR product (amplicons) $1 \mu 1$ was mixed Hi-Di formamide $24.5 \mu 1$ and $5 \mu 1600$ LIZ $^{\mathrm{TM}}$ was used. Electrophoresis was done on the instrument Genetic Analyzer (Applied Biosystem 3500XL). Samples run in the instrument and detail alleles determined using Gene Mapper ID-X software 1.4.

\section{Result and discussion}

Global filer is best and reliable for determination of gender from samples as it contains the 3 markers of Y chromosomes. The problem with male samples were misrepresented as female may be handled in an easy manner especially where quantity of female fraction is comparatively higher than the male or due to deletion of $\mathrm{Y}$ allele in amelogenin marker. In such samples, where mixture of male and female DNA is found and ratio of female is more than 10 times to the male fraction, it becomes very easy to determine the gender of the samples as it contains the $3 \mathrm{Y}$ markers. This kit provides the peaks of other two markers on $\mathrm{Y}$ allele on amelogenin deleted samples. Out of 708 samples, only two males have been identified without $Y$ allele on amelogenin $(.28 \%) .{ }^{8}$ We found that the 2 markers Indel $\mathrm{Y}$ and DYS391, both given the allele 2 and 10 or 11 respectively are common in such Y deletion on amelogenin. The normal male $\mathrm{Y}$ missing profile (AMELY) is given below in Figure 1. While the normal $3 \mathrm{Y}$ markers in global filer kit having allele 2 on Y Indel, XY on amelogenin and 11 on DYS391 is given below in Figure 2.

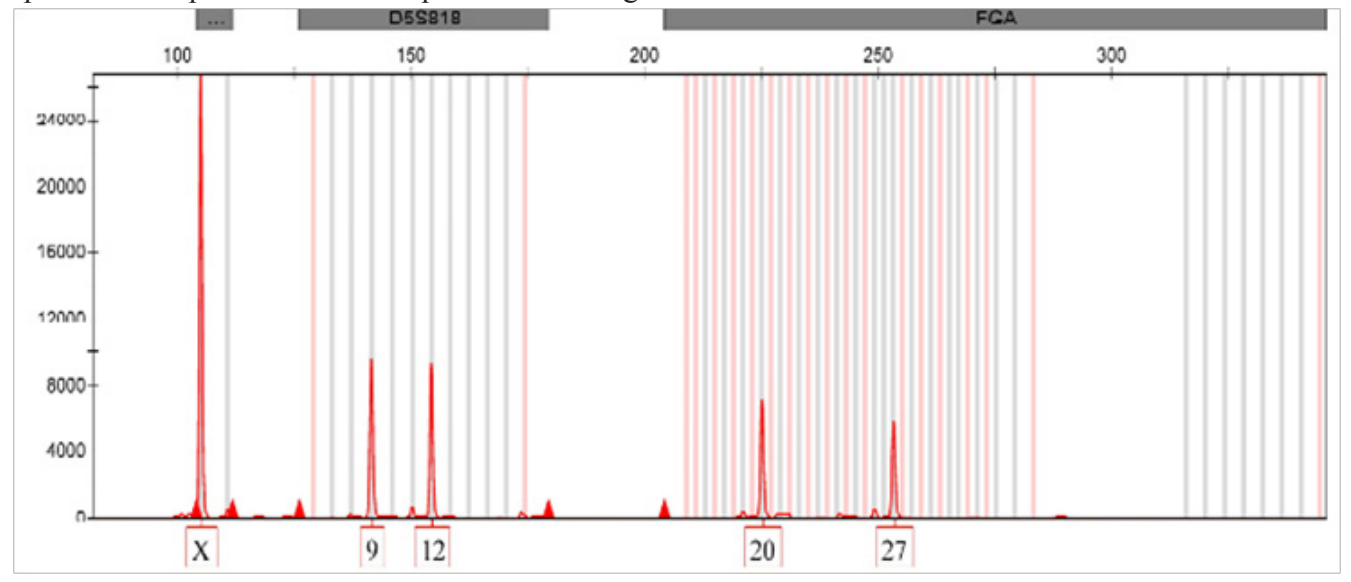

Figure I Normal male Y missing profile (AMELY). 

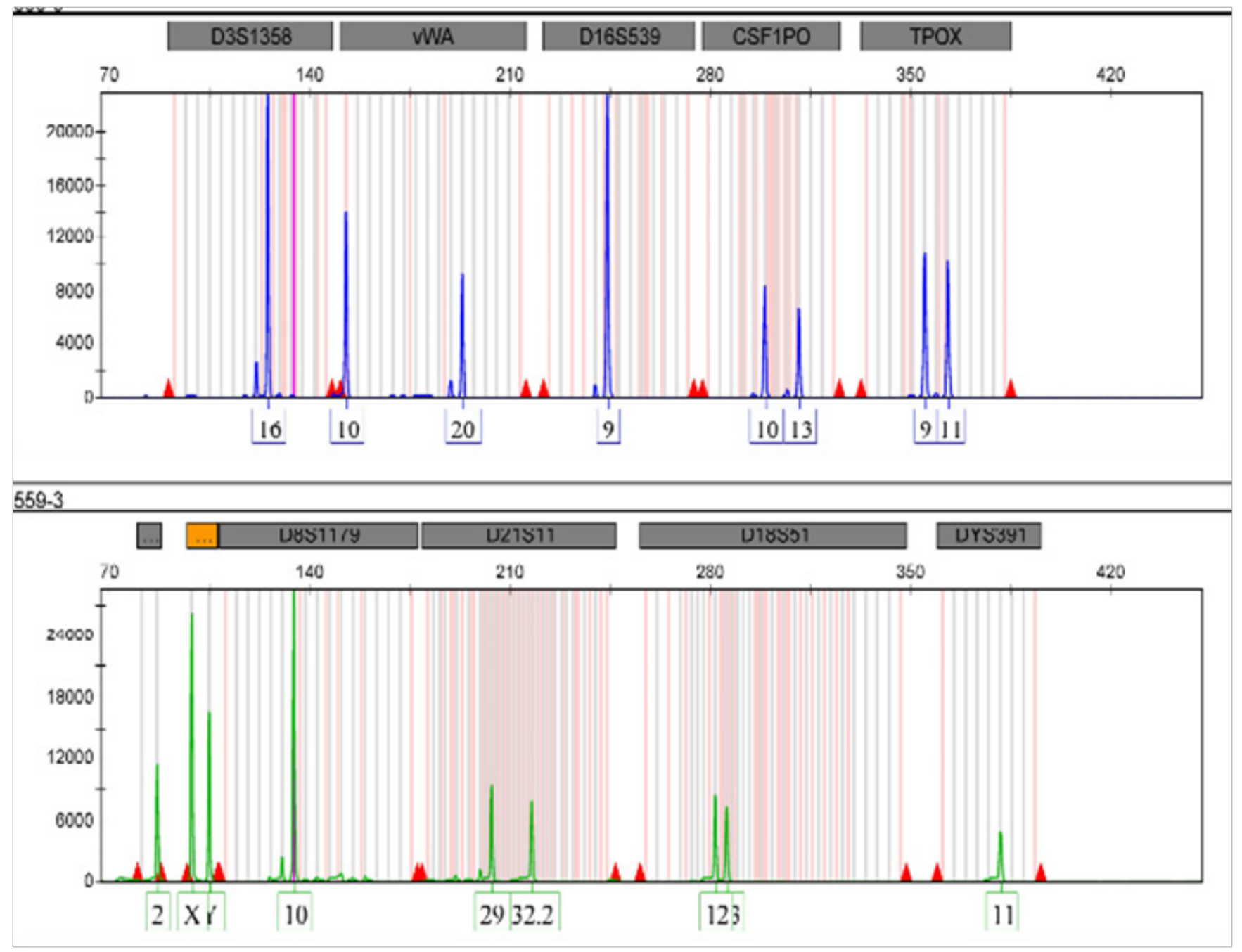

Figure 2 The normal $3 Y$ markers in global filer kit having allele 2 on $Y$ Indel, $X Y$ on amelogenin and I I on DYS39 I.

In addition of the above, in such cases of sexual assault/ rape RTPCR quantitation play an important role for confirmation the gender of a male from the sample. The kit used in quantitation helps to determine the presence of male DNA in the sample. Further Y STR analysis may be used to handle the excess amount of female fraction in the mixed forensic samples. The presence of such male sample can be identified by use of Quantifiler Duo kit of Applied Biosystems on RT PCR machine. If the DNA of a male is confirmed by quantitation from such samples, then it can be further confirmed by using the global filer PCR amplification kit which can amplify 21 autosomal and 3Y STR loci in a single reaction tube. As per the resultant of this study, it is suggested that deletion of $\mathrm{Y}$ allele on amelogenin may be handled with other $2 \mathrm{Y}$ markers also.

\section{Conclusion}

In sexual assault cases or missing identity cases, the determination of gender of DNA sample can be misrepresented during analysis if samples having the only one amelogenin marker. Quantifiler Duo or trio kit is able to ascertain the gender from sample or presence of male in mixed sample in sexual assault cases or other missing identity cases. The obtained result emphasizes the importance of the use of Duo kit and trio kit of of Applied Biosystem in RT-PCR for quantitation as well as for determination of gender. Further, Use of More than 2 markers of Y chromosome will streanthen the case for conclusive opinion, and if there is $\mathrm{Y}$ deletion then the sample is of a male. Precautions should to be taken while preparing the DNA profile of a sample and declaring a result in cases of missing $\mathrm{Y}$ allele in amelogenin. As it may be fatal to the cases, where only amelogenin is used as a sex determinatiing marker. The previous studies have shown the resemblance to this study. Quantity of DNA is not affected in Real Time PCR due to deletion of Y allele on amelogenin. Moreover, less male DNA comparative to female in mixed samples may adversely affect the result negative.

\section{Acknowledgments}

None. 


\section{Conflicts of interest}

The author declares there is no conflicts of interest.

\section{References}

1. Mannucci A, Sullivan KM, Ivanov PL, et al. Forensic application of rapid and quantitative DNA sex test by amilification of the X-Yhomologous gene amelogenin. Int J legal Med. 1994;106(4):190-193.

2. Nakahori $Y$, Hamano K, Iwaya M, et al. Sex identification by polymerase chain reaction using X-Y homologous primers. Am $J$ med genet. 1991;39(4):472-473.

3. Thangaraj K, Reddy AG, Singh L. Is amelogenin gene is reliable for gender identiofication in forensic case work and prenatal diagnosis. Int $J$ legal Med. 2002;116(2):121-123.
4. Laboratory F. National DNA Index system (NDIS) Operational procedure Manual. 2013.

5. Sullivan KM, Mannucci A, Kimpton CP, et al. A rapid and quantitative DNA sex test:Flourescence-based PCR analysis of X-Y homologous gene amelogenin. Biotechniques. 1993;15(4):636-641.

6. Applied Biosystem. 2001.

7. Akane A, Shiono H, Matsubara K, et al. Sex identification of forensic specimen by polymerase chain reaction (PCR) two altenative methods. Forensic Sci Int. 1991;49(1):81-88.

8. Kashyap VK, Sahoo S, Sitalaximi T, et al. Deletion in the Y-derived amelogenin gene fagment in Indian population. BMC Med Genet. 2006;7:37. 